\title{
Electric potential changes prior to shear fracture in dry and saturated rocks
}

\author{
Shingo Yoshida, ${ }^{1}$ Oswald C. Clint, and Peter R. Sammonds ${ }^{2}$ \\ Rock \& Ice Physics Laboratory, Department of Geological Sciences, University College London, London, UK
}

\begin{abstract}
Electric potential changes before shear rupture were measured using Darley Dale sandstone (quartz-rich) and Icelandic basalt (quartz-free) on both dry specimens and in the presence of pore fluid. We find that electric potential changed markedly just prior to dynamic rupture in dry and saturated sandstones and saturated basalt but we did not detect precursory signals in dry basalt. The absence of signals in dry basalt provides strong evidence that the piezoelectric effect and electrokinetic effect are dominant sources for precursory signals. Moreover we find that the amplitude of the precursory signals due to electrokinetic effect in saturated sandstone were as large as the coseismic signals. We propose that this signal is caused by accelerating evolution of dilatancy as cracks grow in the rock before rupture, resulting in water flow into the dilatant region with an electric current produced concurrently.
\end{abstract}

\section{Introduction}

Seismo-electric signals have been used to predict earthquakes in the VAN (after the initials of Varotsos, Alexopoulos, and Nomicos) prediction method in Greece [Varotsos and Alexopoulos, 1984a,b]. There exist high interest and controversy on this method, and the physical origin is not understood [e.g., Geller, 1996]. There have been many laboratory studies on electric signals from rock specimens at the time of fracture, which suggested that the electric signals are produced by the piezoelectric effect of quartz [e.g., Nitsan, 1977], electrokinetic effect due to water movement [e.g., Ishido and Mizutani, 1981], point defects [Varotsos and Alexopoulos, 1986; Hadjicontis and Mavromatou, 1994], emission of electrons [Brady and Rowell, 1986; Enomoto and Hashimoto, 1990], and others. From the view point of earthquake prediction, the generation mechanism of precursory signal is important, but very few experimental results on precursory electric signals have been reported. Here we report measurements of electric potential changes before shear rupture using Darley Dale sandstone (quartz-rich) and Icelandic basalt (quartz-free) on both dry specimens and in the presence of pore fluid.

\section{Piezoelectric Model}

A recently proposed model explains the generation of electric signals due to the piezoelectric effect by stress induced polarization and relaxation [Yoshida et al., 1994; Ikeya and

\footnotetext{
On leave from Earthquake Research Institute, University of Tokyo, Tokyo, Japan.

${ }^{2}$ Also at Greig Fester Centre for Hazard Research, University College London, London UK.
}

Copyright 1998 by the American Geophysical Union.

Paper number 98GL01222.

0094-8534/98/98GL-01222\$05.00
Takaki, 1996; Yoshida et al., 1997]. Quartz in rock subjected to static stress is electrically polarized in proportion to the stress, but the polarization charges are neutralized by compensating bound charges which have moved to the quartz surface (Fig.1(a)). Consequently, the polarization cannot be detected as an electric potential signal from the outside of the rock. When rapid stress change occurs due to dynamic rupture, the neutral state is broken and the difference between the polarization due to the bound charges and the stress-induced piezoelectric polarization appears as an effective polarization, which can be observed from the outside (Fig.1(b)). The polarity of the signal depends on the direction of the electrical axis of the quartz crystals. The effective polarization decays exponentially in the resultant relaxation process with a time constant of $\varepsilon / \sigma$, where $\varepsilon$ is the dielectric constant and $\sigma$ is the electric conductivity (Fig.1(c)). A quantitative model based on this mechanism predicts that the amplitude of the observable signal is proportional to $\varepsilon / \sigma$ times the time derivative of the stress in the case where the time scale of the stress change is longer than $\varepsilon / \sigma$.

\section{Electrokinetic Model}

Electrokinetic phenomena are caused by the presence of an electric double layer formed at the solid-liquid interface. The double layer consists of ions anchored to the solid phase, with an equivalent amount of ionic charge of opposite sign distributed in the liquid phase near the boundary. When the fluid in such a system moves due to pressure gradient, the charges in the fluid are transported in the direction of fluid motion, resulting in electric current. In a porous medium the electric current density $I\left(\mathrm{~A} / \mathrm{m}^{2}\right)$ is described by the following relation [de Groot and Mazur, 1962; Pride, 1994];

$$
I=-\sigma_{f} / F_{1} \operatorname{grad} V+\varepsilon \zeta / \eta F_{2} \operatorname{grad} P
$$

where $\sigma_{f}$ and $\varepsilon$ are the electrical conductivity and the dielectric constant of the fluid, $\zeta$ is the zeta potential (the potential at the slipping plane near the boundary), $\eta$ is the viscosity of the fluid, $P$ is the pressure of the fluid, and $V$ is the streaming potential. $F_{1}$ is the formation factor (defined as the ratio of the conductivity of the fluid to that of the fluid-filled rock), and $F_{2}$ is the formation factor when surface conduction is absent, which are functions of porosity, tortuosity, and hydraulic radius for capillary model [Ishido and Mizutani, 1981]. The first term represents Ohm's law and the second term can be derived by considering a product of charge density (proportional to $\varepsilon \zeta$ ) with flow velocity of the viscous fluid (proportional to $\operatorname{grad} P / \eta$ ). If there are no external current sources, the convection current (due to grad $P$ ) would be balanced by the conduction current (due to grad $V$ ) in the geometry of the laboratory measurements, so

$$
\Delta V=\varepsilon \zeta / \eta \sigma_{f} F_{1} / F_{2} \Delta P
$$

which is the Helmholtz-Smoluchowski equation [Dukhin and Derjaguin, 1974]. The ratio $\Delta V / \Delta P$ is referred to as the 

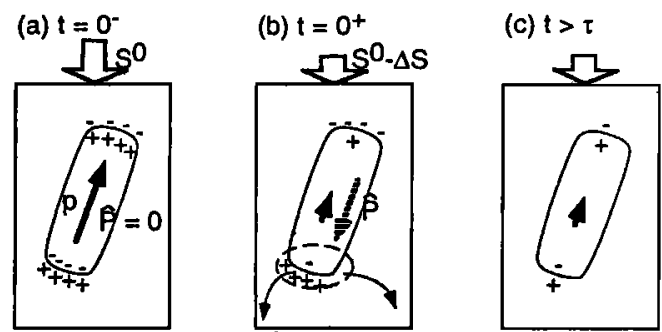

Figure 1. Schematic diagram showing a source model of electric signals based on the piezoelectric effect. (a) Initially, the stress-induced piezoelectric polarization, $p$, is canceled by the compensating bound charges. (b) Rapid stress drop reduces the polarization of the quartz, and the effective polarization, $\hat{P}$, appears. (c) The magnitude of the effective polarization decays exponentially with a relaxation time, $\tau$. (after Yoshida et al., 1997)

streaming potential coefficient. In a general geometry, the divergence of the total current is zero while the zero total current condition may not be satisfied.

This fundamental relation between the streaming potential generated by the fluid moving and the fluid pressure has long been established, however with only this equation we could not predict how an electric signal would result from rock fracture. Many investigators have speculated that dilatancy leads to water movement with an electric current produced concurrently while dilatant crack growth is believed to be important in the generation of seismic precursors [Scholz et al., 1973; Sammonds et al., 1992]. During rock deformation preceding fracture, the streaming potential coefficient should change [Jouniaux and Pozzi, 1995b], and this could also cause electric potential changes. However there has been no direct measurement of electric signals prior to fracture of rocks due to fluid flow change induced by deformation.

\section{Experimental Procedure}

To understand the generation mechanism of seismo-electric signals we have performed triaxial deformation experiments on initially intact specimens of Darley Dale sandstone (quartzrich) and Icelandic basalt (quartz-free), selected to see influence of quartz content and permeability. Darley Dale sandstone is a well indurated feldspathic sandstone with a porosity in the range $12-14 \%$, a permeability of $10^{-15} \mathrm{~m}^{2}$, and a quartz content of about $75 \%$. Icelandic basalt has no quartz, its porosity is 3 $\%$ and permeability is $10^{-20} \mathrm{~m}^{2}$. Rock specimen were air-dried and degassed under vacuum for 24 hours before saturating with water for a week. Cylindrical specimens $40 \mathrm{~mm}$ in diameter by $100 \mathrm{~mm}$ in length were deformed in compression in a highpressure triaxial cell, incorporating a servo-controlled porefluid pressure intensifier and volume monitor. Confining pressure is first applied to the specimen and maintained at a set value. The pore-fluid intensifier piston is advanced to force high-pressure water into the specimen from the upper surface of the specimen and left to equilibrate. For basalt specimens, approximately one day is necessary to achieve saturation. Then an axial load is applied to the rock specimen by a 1400 $\mathrm{kN}$ servo-controlled actuator at a constant displacement rate. During deformation of the specimen the pore-fluid intensifier is set to maintain a constant pore-fluid pressure. The inlet valve was kept open to allow the water to flow between the specimen and the intensifier but the outlet valve of the pipe connected to the bottom surface of the specimen, was closed during the test. Specimens were jacketed in an insulating polymer sleeve as shown in Fig.2. Four electrodes of silver

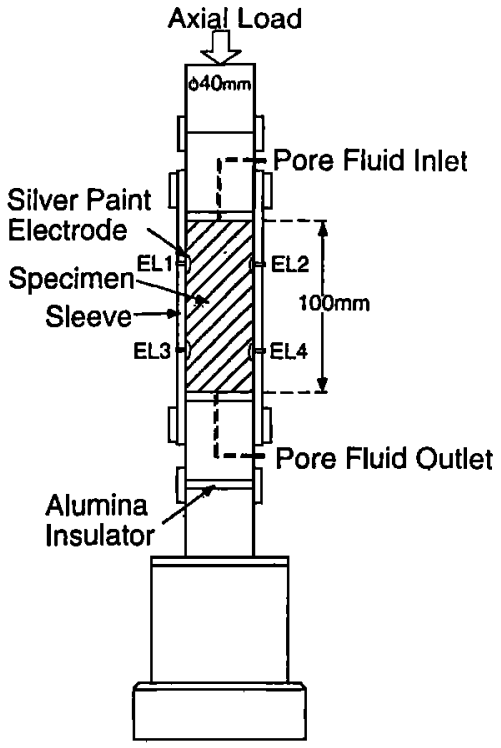

Figure 2. Sample assembly. Four electrodes of silver paint are mounted on the rock specimen $40 \mathrm{~mm}$ in diameter by $100 \mathrm{~mm}$ long.

paint are mounted on the cylindrical surface of the specimen contacting the push-in contact of the sleeve, and then connected to screened co-axial cable. The bottom surface of the specimen is electrically isolated using an alumina plate and also the pipe through which pore water flows is isolated with insulating seal from the surroundings. The electric potential difference between each electrode and the ground is measured using an electronic circuit with high input impedance $\left(10^{13} \Omega\right.$ for two channels, and $10^{15} \Omega$ for other two channels). The differential stress, piston displacement, pore fluid volume and pressure change, electric potential and $\mathrm{AE}$ are recorded continuously during deformation at a sampling frequency up to 1 $\mathrm{kHz}$.

\section{Results}

Figure 3 summarizes our experimental results on sandstone and basalt for both dry and saturated conditions at a strain rate of $1.5 \times 10^{-5} / \mathrm{s}$ and room temperatures. Only the post peak stress, strain softening portion of the stress-time curve is shown, just prior to shear fracture and dynamic stress drop. For dry rock, measurements by the potential meter with an input impedance of $10^{13} \Omega$ are not shown in case they may be influenced by change in resistivity of rock [e.g., Takano et al., 1993]. Figure 4 shows the entire stress curve for saturated sandstone together with the electric potential difference between two electrodes, EL1 and EL3, and the pore fluid movement obtained from the volume monitor of the intensifier. It also shows the time range plotted in Fig.3(b).

Figure 3(a) shows measurements on dry sandstone at a confining pressure of $20 \mathrm{MPa}$. This experiment was done to see how an electric signal is produced by the piezoelectric effect of quartz. Differential stress change and electric potential changes measured at two electrodes, EL1 and EL3, are shown. At about $9 \mathrm{~s}$, dynamic failure occurs with a sudden stress drop. Just before the dynamic event, clear precursory changes in electric potential occurred. The polarities of precursory signal were not the same for the two electrodes; such a feature has also been reported for slip failure [Yoshida et al., 1997]. This 

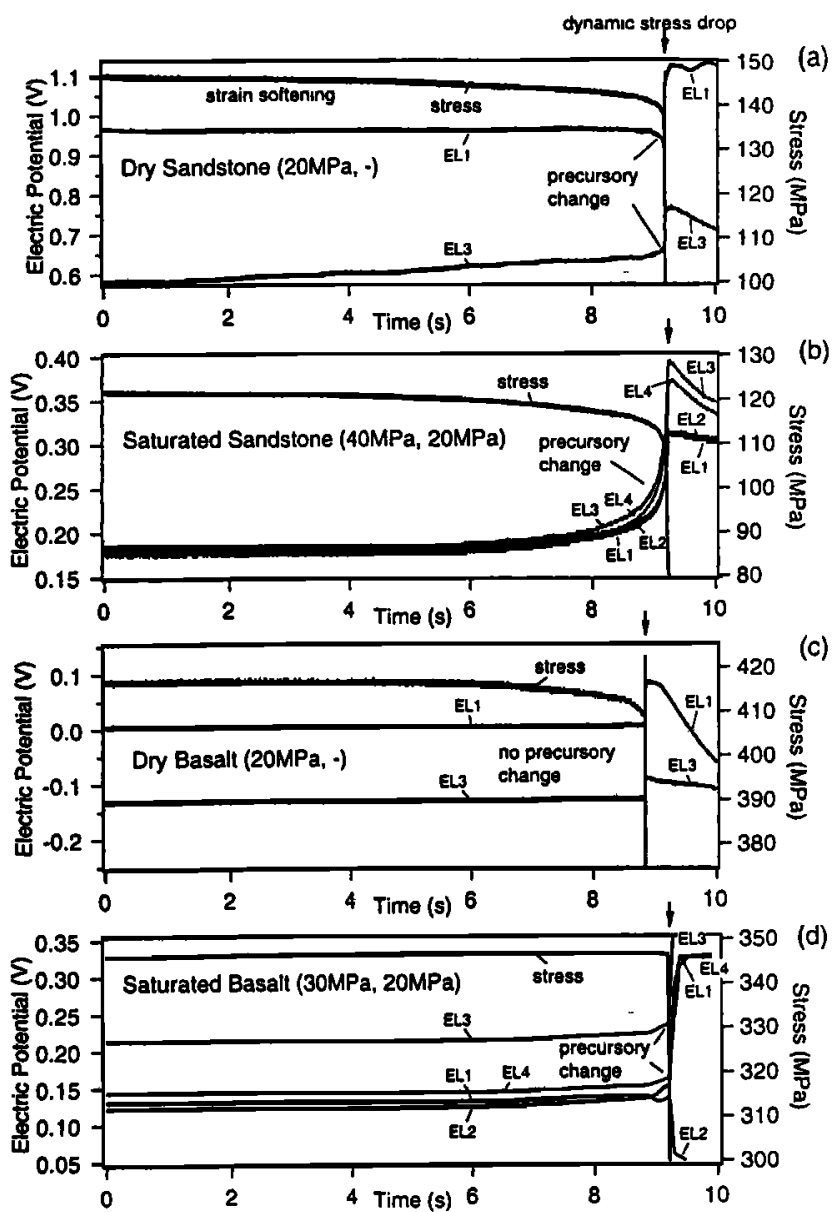

Figure 3. Electric potential changes and differential stress just prior to dynamic failure for dry sandstone (a), saturated sandstone (b), dry basalt (c), and saturated basalt (d). Strain rate is $1.5 \times 10^{-5} / \mathrm{s}$ in all the experiments. The numbers in bracket indicate confining and pore pressures. The precursory electric potential changes occurred just prior to the dynamic failure in dry and saturated sandstones and saturated basalt while no precursory electric signal was found in dry basalt.

is explained by the fact that the precursory electric signal due to the piezoelectric effect is generated in the shear rupture nucleation zone when local stresses are gradually dropping prior to the main rupture. The polarity of the electric signal is governed by orientation of quartz crystal axes which are nearly randomly distributed.

Figure 3(c) shows results from an experiment on a dry basalt specimen for which experimental condition is the same as Fig.3(a). Even though the gradual stress drop is not less than in the other experiments ( $3 a, b$ \& $d$ ), we cannot find precursory changes in electric potential before the dynamic failure. There are many uncertainties about the measurement of electric potential change at the time of the dynamic failure, because artificial noise must have been generated when $\mathrm{AE}$ transducers mounted on the specimen broke. It is possible that basalt generates electric potential change at the failure, but our measurements at that point are contaminated. What we can conclude is that despite a stress change before the dynamic failure no precursory electric signal was generated from dry basalt.

When we used a saturated basalt specimen at a confining pressure of $30 \mathrm{MPa}$ and a pore pressure of $20 \mathrm{MPa}$, we detected clear precursory electric signals as shown in Fig.3(d) despite a small stress change before the dynamic failure. It is obvious

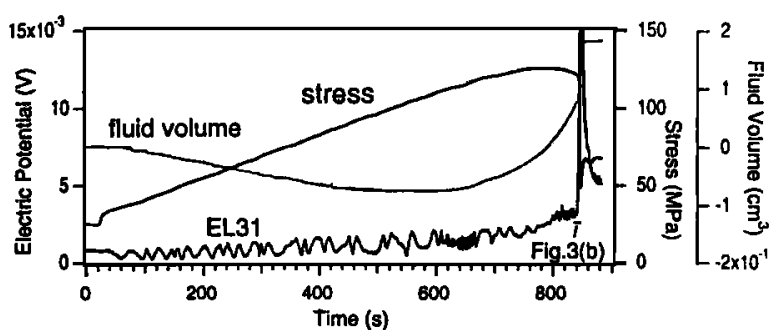

Figure 4. The entire stress curve for saturated sandstone together with the electric potential difference between two electrodes, (EL3 - EL1,) and the pore fluid movement obtained from the volume monitor of the intensifier. It also shows what portion of this curve is plotted in Fig.3(b).

that the precursory signals in the saturated basalt were caused by the presence of pore water because no signal was detected in dry basalt. The absence of detectable precursory signals in dry basalt suggests that other effects than piezoelectric effect and electrokinetic effect are minor sources for generating precursory electric signals.

Results from saturated sandstone at a confining pressure of $40 \mathrm{MPa}$ and a pore pressure of $20 \mathrm{MPa}$, shown in Fig.3(b), demonstrate that the amplitude of the precursory signal can be as large as the coseismic signal. As signals caused by piezoelectric effect are considered to be inversely proportional to electric conductivity as mentioned above, the main source in the saturated sandstone should be the electrokinetic effect. We interpret the high amplitude precursory signal to be generated by transient fluid flow resulting from the accelerating evolution of crack connectivity just before dynamic failure. Some part may be caused by temporal change in the streaming potential coefficient [Jouniaux and Pozzi, 1995b].

Figure 5 shows results of a steady state flow test using a sandstone specimen with an existing fault, which was fractured previously in a triaxial compression test. When the pore fluid outlet valve from the specimen is opened we observe a positive polarity change in electrical potential. During steady fluid flow, until the outlet valve was closed, electric potentials measured at the electrodes were found to be almost constant. This is expected by the theoretical relation (2) because the pore pressure is constant. The potential differences at the lower electrodes, EL3 and EL4, are larger than at the upper electrodes, EL1 and EL2, because the upper surface of the specimen is grounded. When we made the fluid flow in the opposite direction (upward) in a different test, we found the polarity of

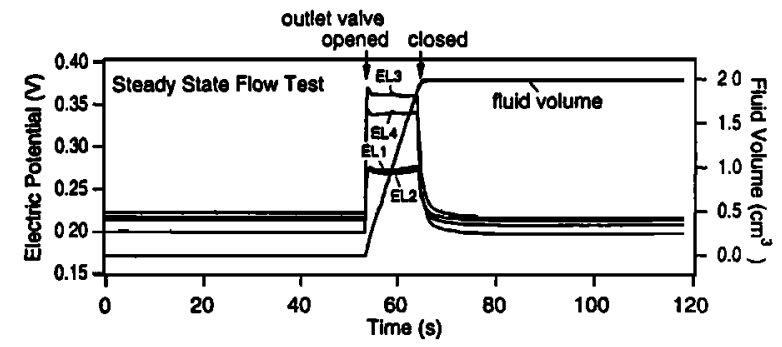

Figure 5. Electric potential changes in a steady state flow experiment on a sandstone specimen. First the pore fluid pressure was kept constant at $20 \mathrm{MPa}$ over the specimen. At $\mathrm{t}=55 \mathrm{~s}$ approximately, the outlet valve was opened resulting in a decrease of the pore pressure to $0.1 \mathrm{MPa}$ at the bottom end of the specimen. Then water started to flow downwards whilst the pore fluid at the top was kept at $20 \mathrm{MPa}$ by the servocontrolled intensifier. The volume of the pore fluid moved into the specimen shows that soon the fluid flow reaches a steady state at a pore pressure difference of about $20 \mathrm{MPa}$. 
the streaming potential was reversed. These polarities are explained by (2) with a negative zeta-potential. From these tests, we have found that the streaming potential coefficient is approximately $-10 \mathrm{mV} / \mathrm{MPa}$ using tap water of $\sigma_{f}=8 \times 10^{-2} \mathrm{~S} / \mathrm{m}$. As Jouniaux and Pozzi [1995a,b] observed, the streaming potential coefficient decreases as the permeability decreases mainly due to the surface conduction effect. Our measurement of $\sigma_{f} \Delta V / \Delta P$ is similar to that measured by Jouniaux and Pozzi [1995b] for Fontainebleau sandstone which has permeability of the same order of magnitude as our specimens i.e. $10^{-15} \mathrm{~m}^{2}$.

Figure 3(b) shows that the polarities of the precursory electric signals in saturated sandstone are positive at all the electrodes. From the streaming potential measurement for steady state flow, we find that positive change occurs when water moves downward. The volume monitor of the intensifier of the pore water showed that about $0.04 \mathrm{~cm}^{3}$ water moved into the specimen from the upper inlet within the final $9 \mathrm{~s}$ before the dynamic failure. Accelerating evolution of dilatancy probably occurs in the whole specimen before dynamic failure, and the main component of flow at every position in the specimen is downward. The fluid volume and the electric potential curves during all the deformation (Fig.4) shows that dilatancy began at about $600 \mathrm{~s}$, and that a slight increase in electric potential appeared to begin about $100 \mathrm{~s}$ after the onset of the dilatancy. The electric potential change was very small (on the order of $\mathrm{mV}$ ) until just before the dynamic rupture, reflecting the fact that the fluid flow rate was very small.

In contrast to sandstone, polarities of precursory signal for saturated basalt varied from electrode to electrode as shown in Fig.3(d). Also we found that the polarities varied depending on individual experiments, which we repeated several times. The volume of the water which has moved into the basalt specimen for the final $9 \mathrm{~s}$ before the dynamic failure was less than $0.0015 \mathrm{~cm}^{3}$, which is much less than for sandstone with 0.04 $\mathrm{cm}^{3}$. For such a short period, the basalt specimen can be viewed as in substantially closed system for water drainage because water cannot reach equilibrium because of the low permeability. Although the amount of dilatancy was not directly measured, it is possible that dilatancy evolves just prior to the dynamic failure, and it causes local fluid movement in the specimen. The directions of local fluid flow, and also convection current, would be a function of position, therefore the polarity of the electric signal would vary depending on the electrode.

\section{Conclusions}

Our experimental results strongly suggest that the piezoelectric effect and electrokinetic effect are the dominant source mechanisms for generating precursory electric signals. Also they demonstrate that the precursory signal due to the electrokinetic effect can be as large as the coseismic signal. Such a feature would not be expected for signal produced by the piezoelectric effect because its amplitude is directly affected by stress, for which the coseismic change is generally much larger than the precursory change. By comparison water movement is only indirectly influenced by stress, therefore under some condition, significant precursory electric signals could be produced by water movement preceding the dynamic failure.

Acknowledgments. We thank J. Bowles, N. Hughes, S. Boon for technical support. We also thank the reviewers $\mathbf{L}$. Jouniaux and $\mathbf{M}$. Takano for useful comments. Financial support for this work was pro- vided by the U.K. Natural Environment Research Council, the Royal Society of London, the Japan Society for the Promotion of Science, and the Japan Ministry of Education, Science and Culture.

\section{References}

Brady, B. T. and G. A. Rowell, Laboratory investigation of the electrodynamics of rock fracture, Nature 321, 488-492, 1986.

de Groot, S. R. and P. Mazur, Non-equilibrium Thermodynamics, NorthHolland, Amsterdam, 1962.

Dukhin, S. S. and D. V. Derjaguin, Surface and Colloid Science, vol. 7, edited by E. Matijevic, John Wiley, New York, 1974.

Enomoto, $Y$. and H. Hashimoto, Emission of charged particles from indentation fracture of rocks, Nature 346, 641-643, 1990.

Geller, R. (ed.), Debate on VAN. Geophys. Res. Lett. 23, No. 11, 1996.

Hadjicontis, V. and C. Mavromatou, Transient electric signals prior to rock failure under uniaxial compression, Geophys. Res. Letr. 21, 1687-1690, 1994

Ikeya, M., and S. Takaki, Electromagnetic fault for earthquake lightning, Jpn. J. Appl. Phys.35, 355-357, 1996.

Ishido, T. and H. Mizutani, Experimental and theoretical basis of electrokinetic phenomena in rock-water systems and its applications to geophysics, J. Geophys. Res. 86, 1763-1775, 1981.

Jouniaux, L. and J. P. Pozzi, Permeability dependence of streaming potential in rocks for various fluid conductivities, Geophys. Res. Lett., 22, 485-488, 1995a.

Jouniaux, L. and J. P. Pozzi, Streaming potential and permeability of saturated sandstones under triaxial stress: Consequences for electrotelluric anomalies prior to earthquakes, J. Geophys. Res. 100, 10,197$10,209,1995 \mathrm{~b}$.

Nitsan, U. Electromagnetic emission accompanying fracture of quartzbearing rocks, Geophys. Res. Lett. 4, 333-336, 1977.

Pride, S. R., Governing equations for the coupled electromagnetics and acoustics of porous media, Phys. Rev. B., 50, 15678-15696, 1994.

Sammonds, P. R., P. G. Meredith. and I. G. Main, Role of pore fluids in the generation of seismic precursors to shear fracture, Nature 359, 228-230, 1992.

Scholz, C. H., L. R. Sykes and Y. P. Aggarwal, Earthquake prediction: A physical basis, Science 181, 803-809, 1973.

Takano, M., I. Yamada and Y. Fukao, Anomalous electrical resistivity of almost dry marble and granite under axial compression, J. Phys. Earth. 41, 337-346, 1993.

Varotsos, P. and K. Alexopoulos, Physical properties of the variation of the electric field of the Earth preceding earthquakes, I, Tectonophysics 110, 73-98, 1984a.

Varotsos, P. and K. Alexopoulos, Physical properties of the variation of the electric field of the Earth preceding earthquakes, II, Determination of epicenter and magnitude, Tectonophysics 110, 99$125,1984 \mathrm{~b}$

Varotsos, P. and K. Alexopoulos, Thermodynamics of point defects and their relation with bulk properties, 474 pp., North-Holland, Amsterdam, 1986

Yoshida, S., P. Manjgaladze, D. Zilpimiani, M. Ohnaka and M. Nakatani, Electromagnetic emissions associated with frictional sliding of rock, in Electromagnetic Phenomena Related to Earthquake Prediction, edited by M. Hayakawa and Y. Fujinawa, pp.307-322, Terrapub, Tokyo, 1994.

Yoshida, S., M. Uyeshima and M. Nakatani, Electric potential changes associated with slip failure of granite: Preseismic and coseismic signals, J. Geophys. Res. 102, 14,883-14,897, 1997.

O. C. Clint and P. R. Sammonds, Rock \& Ice Physics Laboratory, University College London, London WCIE, UK. (e-mail: p.sammonds@ucl.ac.uk)

S. Yoshida, Earthquake Research Institute, University of Tokyo, Tokyo 113-0032, Japan. (e-mail: shingo@eri.u-tokyo.ac.jp)

(Received November 6, 1997; revised March 10, 1998;

accepted April 2, 1998.) 